\title{
CHAOTIC MAP MODELS OF SOOT FLUCTUATIONS IN TURBULENT DIFFUSION FLAMES
}

\author{
S. Mukerji, J. M. McDonough, M. P. Mengüç, S. Manickavasagam \\ Department of Mechanical Engineering, \\ University of Kentucky, Lexington, KY 40506-0108 \\ and \\ S. Chung* \\ Department of Chemical Engineering, \\ University of Illinois, Urbana-Champaign, IL 61801-3792.
}

\begin{abstract}
In this paper, we introduce a methodology to characterize time-dependent soot volume fraction fluctuations in turbulent diffusion flames via chaotic maps. The approach is based on the hypothesis that fluctuations of properties in turbulent flames are deterministic in nature, rather than statistical. Our objective is to develop models of these fluctuations to be used in comprehensive algorithms to study the nature of turbulent flames and the interaction of turbulence with radiation. To this end we measured the time series of soot scattering coefficient in an ethylene diffusion flame from light scattering experiments [1] and fit these data to linear combinations of chaotic maps of the unit interval. Both time series and power spectra can be modeled with reasonable accuracy in this way.
\end{abstract}

\section{INTRODUCTION}

Most practical flames are turbulent in nature. They dissipate their energy predominantly via radiative transfer. These two physical phenomena, along with chemical kinetics and soot formation, need to be understood very well for better control of flames. The nature of each one of these physical mechanisms is very complicated and more importantly, all are coupled. Yet, most of the studies performed over the years have treated each of these independently, and have not considered the interactions among them in detail.

\footnotetext{
"Contributions of S. Chung to this study were part of research conducted at the University of Kentucky as a National Science Foundation Research Experiences for Undergraduates Fellow during the summer of 1996. 


\section{DISCLAIMER}

This report was prepared as an account of work sponsored by an agency of the United States Government. Neither the United States Government nor any agency thereof, nor any of their employees, makes any warranty, express or implied, or assumes any legal liability or responsibility for the accuracy, completeness, or usefulness of any information, apparatus, product, or process disclosed, or represents that its use would not infringe privately owned rights. Reference herein to any specific commercial product, process, or service by trade name, trademark, manufacturer, or otherwise does not necessarily constitute or imply its endorsement, recommendation, or favoring by the United States Government or any agency thereof. The views and opinions of authors expressed herein do not necessarily state or reflect those of the United States Government or any agency thereof. 


\section{DISCLAIMER}

Portions of this document may be illegible in electronic image products. Images are produced from the best available original document. 
Turbulent fluid flow is still not well understood, and radiation transfer is too complicated to be modeled in detail. Furthermore, even if a detailed model is developed, the accuracy of predictions is questionable unless the medium properties are available. These properties, e.g. soot agglomerate size, structure and volume fraction distributions, cannot be predicted with confidence without modeling the interaction of chemical kinetics, turbulence and radiation transfer. In this paper we will outline a formalism with the potential for treating interactions of these phenomena in a computationally efficient, yet realistic, manner.

Detailed discussion of radiation-turbulence interactions in flames is available in the literature in the context of classical Reynolds-averaged-based turbulence models (see e.g., [2]-[7]). Despite the importance of radiation-turbulence interactions throughout the field of combustion, the problem remains "unsolved" in the sense that it is currently not possible to predict flow and heat transfer in high-Reynolds number $(R e)$ combusting systems of practical importance with any degree of confidence. It must first be admitted that modeling of turbulent flows has generally been less than successful in the context of the Reynolds-averaged NavierStokes (N.-S.) equations even in the absence of interactions with other phenomena, and even for quite simple flows, as has been recently demonstrated by Freitas [8]. Fundamental reasons for this have been given by McDonough [9].

If progress is to be made, it will probably have to be in directions different from past efforts. In particular, it is simply not possible to correctly account for time-dependent interactions by using equations that merely model (time-averaged) statistics, even if these equations are highly accurate. This is easily seen by considering prediction of behavior of a flame in an intermittent, gusty flow field. Statistical analysis would be likely to predict preservation of a steady combustion process at some level, whereas the physics of the situation would dictate that sooner or later the flame would be extinguished by a gust, and thereafter there would be no combustion. In light of the obvious importance of understanding and thus being able to control combustion processes, and the fact that past radiation-turbulence interaction analyses have been only partially successful, we have begun studies that represent a distinct departure from more standard modeling techniques.

A new technique, related to chaotic-map models, was theoretically analyzed by Hylin 
and $\mathrm{McDonough}[10]$ in the context of a turbulence modeling procedure known as additive turbulent decomposition (ATD), and the basic ideas associated with the type of model employed have been considered previously in a somewhat ad hoc way by, e.g., Sreenivasan and Ramshankar [11]. The overall ATD procedure consists of solving large-scale, unaveraged governing equations containing small-scale dependent variables that are directly modeled or simulated in some manner closely resembling large eddy simulation (LES). It is important to recognize that this represents a significant departure from the usual Reynolds-averaged methods in which statistics, i. e., correlations of dependent variables, are modeled. It is this feature of modeling physical aspects of the flow that leads to the ability to directly model interactions of turbulence with other phenomena, but at the same time it raises new issues regarding construction of the required models. Methods for carrying out such constructions, and results obtained in a specific case, namely turbulent, radiating flow in a diffusion flame, will be the subject of this paper.

We will present a modeling approach to account for interactions of turbulence with radiation and specifically focus attention on the problem of introducing features of physical measurements into the models. In a recent study, Mengüç et al. [1], a detailed experimental approach was outlined to measure the soot volume fraction fluctuations in a turbulent flame, which were later correlated using chaotic-map models. Even though the results presented in [1] are quite promising, the need for more thorough analysis is obvious. A detailed description of the map construction procedure, per se, in the context of a model problem is presented by McDonough et al. [12].

The key underlying idea is to employ one-dimensional chaotic maps of the unit interval (cf. Collet and Eckmann [13]) as the foundation for the model. The rationale behind this stems from the "universality" properties of certain of such maps, as demonstrated by Feigenbaum [14], and others, and particularly from the fact that their bifurcation sequences can be similar to those of the N.-S. equations (see Pulliam and Vastano [15]). (A non-rigorous plausibility argument for this is given by Frisch [16].) Thus, they appear to provide good candidates for models of fluctuating variables associated with fluid flow and related transport phenomena.

Here, we focus only on the use of chaotic maps for quantification of soot mass fluctuations 
in turbulent diffusion flames. Soot volume fraction plays a major role in radiation-turbulence interactions in flames. In general, soot formation and oxidation processes are controlled by the chemical kinetics, which is a strong function of temperature and species concentration gradients. Both of these gradients are affected by the structure of the turbulent flow field and by strong radiative heating and/or cooling. It will be shown via analysis of experimental results that the radiation-turbulence interactions in sooting diffusion flames can be deterministic in nature, thus motivating the approach being employed here. The main results of this paper are the following: i) a formalism for radiation-turbulence models based on dynamical systems theory, and ii) construction, analysis and comparison with experimental data of such a model specifically for soot volume fraction fluctuations.

\section{CHAOTIC-MAP MODELS}

In this section we will provide details of the approach taken to derive the new types of models described in the Introduction. We begin with a brief outline of the ATD formalism to indicate precisely how such models can be used in the framework of unaveraged governing equations for fluid flow, heat transfer, etc., and thus to provide a rationale for modeling requirements. We then briefly describe the laboratory experiments employed to obtain data for the present study. The reader is referred to [1] for a more complete treatment of the experimental aspects.

\subsection{Additive Turbulent Decomposition}

The modeling procedure we employ here has evolved from early work by McDonough et al. $[17,18]$ and McDonough and Bywater $[19,20]$ on "large-scale/small-scale decomposition" approaches applied to the Burgers' equation model of the N.-S. equations. Additive turbulent decomposition is now viewed as a family of methods ranging from highly-parallelizable direct numerical simulation (DNS) (cf. McDonough and Wang [21] and Mukerji and McDonough [22]) to the very efficient modeling procedures introduced in [10], and employed in McDonough et al. [23, 24] and Salazar et al. [25]. It is this latter form of ATD to which the 
present chaotic map construction pertains.

The ATD formalism consists of unaveraged, time-dependent transport equations along with an additive decomposition of both the dependent variables (as in large-eddy simulation) and of the governing equations themselves. We note that the nonlinear Galerkin (NLG) procedures of Temam and various co-workers (e.g. Marion and Temam [26]) comprise a special case of ATD, as is clear from recent work of Brown et al. [27]. If we let q denote the dependent variable vector, then in the above context we can write

$$
\mathbf{q}(\mathbf{x}, t)=\sum_{k=1}^{K} \mathbf{q}^{(k)}(\mathbf{x}, t), \quad \mathbf{x} \in \mathbb{R}^{d}, d=1,2,3,
$$

where each $\mathrm{q}^{(k)}$ represents a "scale of behavior" of the physical phenomena (or, in mathematical terms, an element of a subspace of an appropriate Hilbert space), and $\mathbb{R}^{d}$ denotes $d$-dimensional Euclidean space. When $K=2$ we have

$$
\mathbf{q}(\mathbf{x}, t)=\overline{\mathbf{q}}(\mathbf{x}, t)+\mathbf{q}^{*}(\mathbf{x}, t)
$$

which is analogous to LES if we view $\overline{\mathbf{q}}$ as the "resolved" scales and $\mathbf{q}^{*}$ as the "unresolved" scales.

A typical transport equation for $q$ can be expressed in the general form

$$
\mathbf{q}_{t}+\nabla \cdot \mathbf{F}(\mathbf{q})=\nabla \cdot \mathbf{G}(\mathbf{q})+\mathbf{S}(\mathbf{q})
$$

where $\mathbf{F}, \mathbf{G}$ and $\mathbf{S}$ are advective (nonlinear), diffusive and source terms, and the subscript $t$ denotes differentiation with respect to time; $\nabla$ is the gradient operator. Substitution of (2) into (3) results in

$$
\left(\overline{\mathbf{q}}+\mathbf{q}^{*}\right)_{t}+\nabla \cdot \mathbf{F}\left(\overline{\mathbf{q}}+\mathbf{q}^{*}\right)=\nabla \cdot \mathbf{G}\left(\overline{\mathbf{q}}+\mathbf{q}^{*}\right)+\mathbf{S}\left(\overline{\mathbf{q}}+\mathbf{q}^{*}\right)
$$

We observe that in typical Reynolds-averaged approaches Eq. (4) would be averaged, whereas in "complete" ATD it would be additively decomposed into large- and small-scale parts (see [21] for details).

In the present case we would solve (4) for $\overline{\mathbf{q}}$, and model $\mathbf{q}^{*}$. This leads to efficient computational procedures that can be applied on coarse grids (fine meshes are not needed 
to resolve large-scale behavior), but which are capable of predicting small-scale flow features such as transition to turbulence, relaminarization and interactions between fluid motion and other physical phenomena. It is the construction of these models, especially the fluctuating chaotic maps on which they are based, that comprises the main focus of the present work.

\subsection{General Form of $\mathbf{q}^{*}$}

As implied above, we expect to solve Eq. (4) on relatively coarse grids (generally no finer than might be used in Reynolds-averaged method solutions) intended to accurately resolve only the large scales of motion. In particular, it should not be expected that $\overline{\mathbf{q}}$ will include any significant portion of inertial subrange scales. It follows that $\mathbf{q}^{*}$ must serve as a correction to this under-resolved solution approximation, and must in some sense model a significant portion of the effects of energy transfer through the inertial subrange, and the dissipation of energy at the Kolmogorov scales.

In principle there are at least several ways by which one might accomplish this, and here we describe only the approach we believe to be the simplest. Before providing the details, however, we note a fundamental constraint that we wish to impose on any such model. Namely, we require $\mathbf{q}^{*} \rightarrow \mathbf{0}$ as discretization step sizes go to zero in order to preserve the consistency of (4) with (3). The obvious consequence of this is that for this class of models, the complete procedure automatically collapses to DNS. We comment that the rate at which this occurs with respect to refinement of the discretization is also very important for accuracy; this is treated in [10].

We must also emphasize that the quantities being modeled here are distinctly different from those modeled in Reynolds-averaged approaches and in LES: $\mathbf{q}^{*}$ is the vector of fluctuating dependent variables rather than statistical correlations between various combinations of these. This leads to several advantages, the most important of which are the ability to model instant-by-instant fluctuations and interactions, the significant reduction in the number of quantities requiring a model, and the ability to obtain direct laboratory measurements for all modeled quantities. The first of these addresses problems associated with highly unsteady flows alluded to in the Introduction. The second leads to greatly simplified 
modeling procedures; the reader is referred to Wilcox [28] to gain an appreciation for the extreme complexity of modern Reynolds-averaged-based models. This will also provide an understanding of the experimental difficulties associated with such models; viz, the models require experimental results for numerous statistical properties that cannot be measured, and often cannot even be accurately deduced from measurements.

The form of the chaotic map model considered here is

$$
\mathbf{q}^{*}=\mathbf{A} \zeta \mathbf{M}
$$

where $\mathbf{A}$ is an amplitude factor constructed from classical theories of isotropic turbulence (cf. Tennekes and Lumley [29]); $\zeta$ is an anisotropy correction factor consisting mainly of first-order structure functions of (local in space and time) high-pass filtered resolved-scale variables, and $\mathbf{M}$ is a vector of nonlinear (potentially chaotic) algebraic maps. The amplitude factor must account for scales of motion ranging from just smaller than the resolved scales to the Kolmogorov scales, and it may be different for different components of $\mathbf{q}^{*}$. The anisotropy factor must account for both physical and numerical grid anisotropies, the latter of which have proven difficult to treat in the context of LES specifically because of filtering used to obtain equations for the resolved scales. Finally, the chaotic map $\mathbf{M}$, the main topic of the present study, must reflect the bifurcation sequences, time scales and general fluctuating character of physical variables over the appropriate (as induced by the resolved-

scale calculations) range of scales. A complete analysis of Eq. (5) is provided in [10], and a more detailed summary than presented here (with formulas for $\mathbf{A}$ and $\zeta$ ) can be found in [23]. In neither of these references, however, is much concern given to choice of the map $\mathbf{M}$, or to its construction from experimental data. We will address these issues in Sec. 3 .

\subsection{Experimental Data}

Experiments were performed using a coflow diffusion flame burner. The burner consists of a central fuel tube surrounded by a co-annular oxidizer tube having diameters of 1 and $5 \mathrm{~cm}$ with an overall height of $35 \mathrm{~cm}$, and has been designed to produce a flat velocity profile of the oxidizer and the fuel. The oxidizer tube has two diametrically opposed inlet ports which make possible an even distribution of the oxidizer across the cross-section of the tube. 
As oxidizer, pressurized bottled air was used. Ethylene ( $99.5 \%$ purity) was employed as fuel. A diagnostic system based on a short-pulse, $532 \mathrm{~nm}$ wavelength, solid-state Q-switched Nd:YAG laser with an optical parametric oscillator was utilized for data collection. More details of this experimental system are available in [1].

The underlying intent of the experiments conducted for this study is to obtain data on angularly scattered light intensity as a function of time. This variation in recorded intensity is related to transience of the scattering particles (soot) in the control volume at the focal point of the laser beam. Experiments were conducted to obtain data on scattered light intensity from soot particles at $30^{\circ}$, providing a measure of soot volume fraction, $f_{v}$, as a function of time. The time series were obtained by making single-shot measurements at the rate of thirty data points per second (i. e., at laser pulse frequency) for the ethylene flame using several different air-fuel ratios, and measurements were recorded at several different heights.

It is important to realize that similar experiments can be carried out with a continuouswave laser modulated using an optical chopper. These types of experiments are needed to identify the effects of data collection frequency on observables. As discussed before, however, our objective here is to present a methodology rather than bringing a complete solution to the problem. For this reason we did not conduct experiments other than those performed at a single frequency of $30 \mathrm{~Hz}$.

The Reynolds number of the flame was predicted to be approximately $R e=400 \mathrm{~L}$, where $L$ is the flame height as measured (in $\mathrm{cm}$ ) from the nozzle tip. These Reynolds numbers are not very large; thus, the flame itself is, to an extent, in the buyoncy-influenced regime. This provides a possible explanation for observed strong oscillations at about $12 \mathrm{~Hz}$, which may be due to the buoyant instability as discussed in [30]. With increasing Reynolds numbers, this instability is likely to lose its importance. Indeed, our experimental data also show that with increasing flame height, the importance of $12 \mathrm{~Hz}$ oscillations diminishes.

The fundamental hypothesis in our approach to analyzing these data, and subsequently constructing turbulence models, is that turbulent fluid flows and any associated interacting phenomena are deterministic, rather than stochastic, in nature. It should be noted that 
this hypothesis is amply supported by both theoretical and experimental results beginning with the work of Ruelle and Takens [31]. It is therefore reasonable to employ deterministic dynamical systems as turbulence models, and we remark that the N.-S. equations comprise one such system. But there exist much simpler systems possessing many of the features of the N.-S. equations, viz., chaotic time series and bifurcation sequences leading from steady to periodic, and on to chaotic behavior.

There are a number of important tools that have been developed for the qualitative and quantitative analysis of dynamical systems, or more precisely, for analysis of time series generated by the evolution of such systems. The reader is referred to Guckenheimer and Holmes [32] and Bergé et al. [33] for very readable treatments. Here, we will specifically employ the following in the analysis of our experimental data: i) time series, ii) power spectral density (psd), iii) delay maps, and iv) various statistical characterizations; but we caution that use of additional ones such as Poincare maps, correlation dimension, etc. may be necessary to obtain more refined results. Moreover, it should be emphasized that none of these quantities (i. e., power spectra, fractal dimension, etc.) can be inverted to imply a unique time series. Thus the modeling effort must employ more sophisticated analyses and quantification of the time series behavior itself, e. g., use of total variation per unit time, frequency of zero crossings, etc., in order to more precisely model complex, aperiodic deterministic phenomena. These latter techniques have recently been employed in [12] in the context of fitting data obtained from a model problem. We will utilize several of the most effective techniques identified in that study below. Also note that in our earlier study, [1], the modeling effort was limited to fits of psd profiles.

We begin with direct observation of time series of measured data in an effort to detect general features of the temporal behavior of the phenomenon under consideration such as periodicity, intermittency or more complex behavior. Comparison of time series from different spatial locations and/or for different values of a control (bifurcation) parameter, e. g., air-fuel ratio, may reveal features suggesting a particular bifurcation sequence, and thus a particular chaotic map (or maps) to be employed as a model.

Figure 1 presents four snap-shots of the flame over a one second time interval. The 
exposure for each image is $1 / 4000$ seconds. The base diameter of the flame is $1 \mathrm{~cm}$. The Reynolds number of the flame is increasing with increasing height, and for the flame depicted it can be given roughly as $R e=400 L$, where $L$ is the length measured from the nozzle (in $\mathrm{cm}$ ). The corresponding time series and psd at several axial locations are shown in Figs. 2(a-c) and $3(\mathrm{a}-\mathrm{c})$, respectively. The time series suggest that the flow becomes more turbulent at higher axial locations (increasing $R e$ ). In particular, these data are strongly suggestive of a subharmonic bifurcation sequence of the Feigenbaum type (see [14]). As will be discussed in more detail in the next section, it is possible to gain considerable information regarding time scales and amplitudes of various flow "structures" simply through careful qualitative analysis of the time series. But if details regarding periodic, subharmonic or quasi-periodic features are required (as they are for our present purposes), it is necessary to calculate the power spectra. We comment that for turbulent, chaotic data it is typical for the psd to consist of a broad-band spectrum (significant power over a wide range of frequencies) with $f^{-\alpha}$ decay, with $\alpha$ often in the range $0<\alpha<2$. The psds displayed in Fig. 3(a-c) show only a limited decay, but on the other hand there is clear evidence of a subharmonic bifurcation sequence (the $6 \mathrm{~Hz}$ and $12 \mathrm{~Hz}$ peaks in Fig. 3(b,c)). Thus, the chaotic map(s) chosen for the modeling process should at least exhibit this feature. (Here, it is worth to noting that the $12 \mathrm{~Hz}$ oscillations are in the range of those reported by Buckmaster and Peters [30].)

\section{MODEL CONSTRUCTION}

We begin model construction by noting that the power spectra presented in Fig. 3(a-c) are very similar to those obtained from the time series of the logistic map, a widely studied nonlinear algebraic map (cf. [13]). Such strong similarities earlier motivated us to employ a slightly modified form of the logistic map,

$$
m_{k}^{(n+1)}=b_{k} m_{k}^{(n)}\left(1-\left|m_{k}^{(n)}\right|\right)
$$

as a simple model for fluctuating absorption coefficient [24]. Here, $b_{k}$ is the bifurcation parameter (analogous to $R e$ in the N.-S. equations) and $m_{k}^{(n)}$ is the map iterate at the $n^{\text {th }}$ iteration level. Since we will be attempting to fit experimental data using a combination of 
maps of the form (6), we use an index $k$ to distinguish different maps in the combination. Equation (6) has also been used in [1], [12] and [23].

Although there are strong similarities between the experimental results and those obtained from the logistic map, there are also obvious differences in detail. For the present experimental data the psds clearly show at least three distinct time scales in the spatially lower part of the flame in addition to the broad-band behavior, and a simple logistic map such as Eq. (6) is not able to replicate this. There are numerous means by which this may be addressed. Here we will begin with a simple linear combination of logistic maps of the form (6):

$$
M^{*}=\sum_{k=1}^{K} \alpha_{k} \mathcal{S}_{k}^{(n+1)}\left(\omega_{k}, d_{k}, m_{k}\left(b_{k}\right)\right)
$$

where the superscript $(n+1)$ indicates an advanced iteration level. Each $m_{k}^{(n)}$ in Eq. (7) is calculated from Eq. (6), but with different values of $b_{k}$. These values are employed in a function, $\mathcal{S}_{k}^{(n)}$, defined below, and the results for each index $k$ are weighted by $\alpha_{k}$. The final value of the map combination at the advanced iteration is constructed by adding a "history" term so that the complete map takes the form

$$
M^{(n+1)}=\theta M^{*}+(1-\theta) M^{(n)} .
$$

Here $\theta$ is an "implicitness" factor which determines the weight assigned to the history term.

The coefficient $\mathcal{S}_{k}^{(n+1)}$ in Eq. (7) depends on the frequency of evaluation, $\omega_{k}$, and the duration, $d_{k}$, over which each map remains active once it is switched on. This coefficient depends on a switching function, $\mathcal{F}_{k}^{(n)}$, and a duration function, $\mathcal{D}_{k}^{(n)}$. The first of these is defined as

$$
\mathcal{F}_{k}^{(n)} \equiv \begin{cases}1 & \text { if }\left(n \bmod \omega_{k}\right)=0 \\ 0 & \text { otherwise }\end{cases}
$$

This determines whether the $k^{\text {th }}$ map is to be activated at the $n^{\text {th }}$ iteration. The duration 
function is defined as

$$
\mathcal{D}_{k}^{(n)} \equiv \begin{cases}1 & \text { if } \mathcal{F}_{k}^{(n)}=1 \\ \mathcal{D}_{k}^{(n-1)}+1 & \text { if } \mathcal{F}_{k}^{(n)}=0 \text { and } 0<\mathcal{D}_{k}^{(n-1)}<d_{k} \\ 0 & \text { otherwise. }\end{cases}
$$

This function determines how long a map remains active. It is initialized to 1 when the map is activated according to equation (9), is incremented by 1 each time the map is evaluated and is set to 0 if it exceeds $d_{k}$. The switching function, $\mathcal{F}_{k}^{(n)}$, is evaluated at every iteration independent of $\mathcal{D}_{k}^{(n)}$. Whenever $\mathcal{F}_{k}^{(n)}$ is unity $\mathcal{D}_{k}^{(n)}$ is initialized to 1 , and the $k^{\text {th }}$ map is (re-)activated. This happens irrespective of the current value of $\mathcal{D}_{k}^{(n)}$. Based on these function definitions, the coefficient, $\mathcal{S}_{k}^{(n)}$, is computed as

$$
\mathcal{S}_{k}^{(n)}= \begin{cases}m_{k}^{(n-1)} & \text { if } \mathcal{D}_{k}^{(n)}=0 \\ m_{k}^{(n)} & \text { otherwise }\end{cases}
$$

Our goal in the present research is to determine $b_{k}, \alpha_{k}, \omega_{k}$ and $d_{k}$ so as to best fit the experimental time series with the time series obtained from the chaotic map model, $M^{(n)}$.

\subsection{Data-Fitting Criteria}

In this subsection we present one possible approach based on a least-squares minimization of differences between various properties of measured and modeled time series. We will briefly discuss the procedures successfully used for model problem data in [12] now applied to the experimental data sets shown in Fig. 2(a-c).

The first step in this procedure is to select properties of the time series to quantify differences between measured and modeled results. We should emphasize that the set of properties employed here is not unique, but it appears to be reasonably effective. It includes both mathematical and physical characterizations: averages, total variation, $L^{1}$ and $L^{2}$ norms, slope sign changes per unit time, global minima and maxima, mean crossings per unit time, skewness, flatness, autocorrelation and "extended" intermittency factors. We discuss each of these briefly in what follows and provide specific formulas used in our analyses. More details can be found in [12]. 
One of the most basic quantifications of a time series that should match between measured and modeled results is the time average, $\bar{u}$. But given the complicated nature of the time series shown in Fig. 2(a-c), we must refine the analysis by measuring the separate averages of the positive and negative values of $u$, denoted $\bar{u}^{+}$, and $\bar{u}^{-}$, repectively. The other gross quantifications of the data which we monitor are $u_{m a x}^{+}, u_{\min }^{+}, u_{m a x}^{-}$and $u_{\min }^{-}$the maximum and minimum of positive and negative values of $u$.

In addition to the averages, we also measure the average variation of the time series,an important quantification of oscillatory behavior. As was done before for the averages, we will employ separate positive and negative variations. We also measure the number of zero crossings per unit time, $N_{0}$, number of positive mean crossings, $N_{\text {meant }}$ and number of negative mean crossings, $N_{\text {mean- }}$, as an additional step towards matching oscillation frequencies between modeled and measured data. This can also be quantified by the number of changes in sign of the slope between successive pairs of points in the time series normalized by the total number of points, $N_{S^{ \pm}} / N$, denoted $f_{s^{ \pm}}$. Just as was done for variation of the time series, we can separately consider this fraction for the positive and negative parts $f_{s^{ \pm}}^{+}$and $f_{s^{ \pm}}^{-}$.

The final property of the time series to be considered here that directly involves detailed oscillatory behavior is what we will term the intermittency distribution. Recall that the intermittency factor, or simply intermittency, at a point in a turbulent flow is the fraction of time during which the flow is turbulent at that point. Here we will define intermittency levels by first requiring that the segment of the time series under consideration be oscillatory, and then establishing the amplitude range of the oscillations as a fraction of the interval $\left[u_{m i n}, u_{m a x}\right]$. The number of intermittency levels employed will be specified at the time analysis of a given data set is initiated, and can be expected to change somewhat from one time series to the next. Because the intermittency distribution proved to be one of the most important measures of oscillatory behavior, and because to our knowledge it has not previously been used for analysis of experimental data, we will treat it in more detail.

To check whether a given segment of data is oscillatory we carry out the following test. 
At the $n^{\text {th }}$ discrete point we calculate the forward and backward differences,

$$
\begin{aligned}
& \Delta_{+} u^{(n)} \equiv u^{(n+1)}-u^{(n)}, \\
& \Delta_{-} u^{(n)} \equiv u^{(n)}-u^{(n-1)} .
\end{aligned}
$$

If either of the following inequalities is satisfied we consider the time series to be oscillatory in a neighborhood of the $n^{\text {th }}$ point:

$$
\begin{aligned}
\left(\Delta_{+} u^{(n)}\right)\left(\Delta_{-} u^{(n)}\right) & <0, \\
\left(\Delta_{+} u^{(n)}\right)\left(\Delta_{-} u^{(n-1)}\right) & <0 .
\end{aligned}
$$

Once it has been established that a point is part of an oscillation it is then possible to assign it to an intermittency level. We denote the intermittency levels by $I_{i}$ and the end points of these levels by $g_{i}$. Then for a total of $M$ intermittency levels $\left(I_{1}, \ldots, I_{M}\right)$ there are $M+1$ end points $\left(g_{0}, \ldots, g_{M}\right)$ with $g_{0}=u_{\min }$ and $g_{M}=u_{m a x}$. The intermittency levels can now be defined in terms of $g_{i}$ s as:

$$
\begin{aligned}
I_{1} & =\left[g_{0}, g_{1}\right) \\
I_{2} & =\left[g_{1}, g_{2}\right) \\
\vdots & \\
I_{M} & =\left[g_{M-1}, g_{M}\right] .
\end{aligned}
$$

We will denote the number of points of the time series in an intermittency level $i$ by $N_{I_{i}}$. We can then define the intermittency factors for each level as

$$
f_{I_{i}}=N_{I_{i}} / N
$$

The choice of $M$ and the spacing between the $g_{i}$ s depends on the type of intermittency information that is critical to the analysis and has to be matched by the modeled time series.

In addition to the measures already discussed, we also calculate some global properties which may be necessary for production of accurate models. A global mathematical quantity that is widely used to characterize the "size" of functions is the norm, $\|\cdot\|$. In this study we have employed the time averaged $L^{1}$ and $L^{2}$ norms, defined in the usual way. From a 
purely mathematical standpoint the $L^{1}$ norm is sometimes more appropriate, while the $L^{2}$ norm has the physical interpretation of energy.

There are additional statistical properties that are widely used in studies of turbulence, which may be appropriate quantifiers of the time series we study here. These include the autocorrelation function, the probability density function (pdf), the flatness $F$ and the skewness $S$. The definitions for these are well known and can be found, for example, in [29].

\subsection{Least-Squares Functional}

The various measures we have discussed for quantifying data in the form of time series will be used in the model construction process. If we let $p_{i}^{\text {meas }}$ and $p_{i}^{\text {model }}$ denote the numerical value of property $i$ as obtained from experiment and model, respectively, and define

$$
\delta p_{i} \equiv p_{i}^{\text {meas }}-p_{i}^{\text {model }}
$$

then we can define a weighted least-squares functional corresponding to $N_{p}$ properties as

$$
Q(\alpha, b, \omega, d)=\sum_{i=1}^{N_{p}} \phi_{i}\left(\delta p_{i}\right)^{2},
$$

where $\alpha=\left(\alpha_{1}, \alpha_{2}, \ldots, \alpha_{k}\right)^{T}$, etc., and $\phi_{i}$ are the weights associated with different properties. We can then determine the unknown amplitudes, bifurcation parameters, frequencies and durations of map evaluations by minimizing $Q$. Conceptually this is straightforward, but in practice it is difficult in the present case because the $\delta p_{i}$ are generally not differentiable with respect to $b, \omega$ and $d$. Thus, direct search techniques have been used in the present study. We comment that the use of neural networks or simulated annealing techniques could be very effective in these searches, and these will be investigated in future studies.

\section{RESULTS AND DISCUSSION}

In this section we present results from applying the analysis techniques discussed in the preceding section to the experimental time series of scattered light intensities from soot particles obtained from laboratory experiments described in Section 2.3. The data fitting 
approach directly yields the parameters of the map combination which produce the best fits to the time series data at three separate axial locations along the flame. Thereafter, we use the map combination, with parameters obtained by interpolation, to predict the time series at intermediate locations in the flame. This is important because in the intended use of these maps as subgrid-scale models, we expect to specify the parameters of only a few maps at discrete spatial locations in the flow by data fitting while the parameters for maps at the intermediate locations have to be obtained by interpolation.

All results to be presented were computed in double precision (64-bit) FORTRAN on the HP-Convex Exemplar SPP 1200 at the National Center for Supercomputing Applications, University of Illinois, Urbana-Champaign.

\subsection{Results of Data-Fitting}

The experimental time series at different axial locations along the flame are shown in Fig. 2(ac). The first 5000 time steps in the time series were neglected to remove the effect of initial transients from the data. The subsequent 10000 time steps were used for data-fitting computations to guarantee a sufficiently long stationary state to permit reliable analyses of the sort being performed here. It should be noted that only a small segment, spanning 600 time steps, of the complete time series is shown in Fig. 2 so that salient features can be clearly identified. The corresponding power spectra and delay maps are shown in Figs. 3(a-c) and $4(\mathrm{a}-\mathrm{c})$, respectively. The power spectrum of the experimental time series was computed using the last 8192 data points, and the delay map was constructed based on the last 2000 points with a delay of 4 . The previously discussed measures for quantifying time series data were computed for each of the experimental time series of Fig. 2(a-c) and are shown in the third column of Table 1 for only the $h=4.0 \mathrm{~cm}$. axial location. These measures include intermittency factors from Eq. (15) in 10 equally spaced intervals, i.e. $M=10$ in Eq. (14). These intermittency factors are also shown as histograms in Fig. 5(a-c).

The time series show evidence of at least three different scales of structure in both space and time, suggesting that initially we should attempt a fit with three terms $(K=3)$ in the linear combination of logistic maps (7). The evidence of enhanced turbulence levels at higher 
axial locations is clear as the oscillation frequencies and amplitudes of the time series increase going from Fig. 2(a) to 2(c). Evidence of this increasing turbulent activity going up the flame can also be seen in the psds where the overall power levels increase from Fig. 3(a) to 3(c). But the most striking feature to be noted in the power spectra are the wide peaks at $6 \mathrm{~Hz}$ and $12 \mathrm{~Hz}$ in Figs. 3(b) and 3(c). These peaks occur at lesser power levels in Fig. 3(a). This is clear evidence of a subharmonic bifurcation. Also present in each psd is a sharp peak at $10.8 \mathrm{~Hz}$, possibly the result of quasi-periodicity.

Delay maps (shown in Fig. 4) seldom provide anything more than a qualitative indication of topology of a dynamical system, but they provide a simple (though not completely foolproof) indicator of deterministic, as opposed to random behavior. In this case it can be seen that the delay map of Fig. 4(c) is more "space-filling" than that of 4(a) yielding further evidence of increased turbulent behavior.

The detailed data-fitting procedure is carried out for each of the three experimental time series shown in Fig. 2(a-c). The first step in the analyses is to set up a parameter space by choosing a wide range of combinations of values of $\alpha_{k}, b_{k}, d_{k}$ and $\omega_{k}, k=1,2,3$. Each chaotic map combination is characterized by 12 parameters which are selected as unique permutations of values in the parameter space. The time series of the chaotic map combination is generated for each of these permutations using equations (7) and (8). The same quantifying statistics that were calculated for the experimental data are now computed for each model time series, and the least-squares functional, Eq. (17), is then constructed for each case. The computations for each case are independent of all others and are done in parallel. A direct search is used to identify the cases with small values of the functional, $Q$. These cases are considered to be the 'best' cases, and ten of these cases are stored in each run. These results are used to manipulate the parameter space for the subsequent runs. Initially, coarse-grained searches are made on slightly overlapping segments of the parameter space. Once the best segment has been identified, fine-grained searches are made on smaller subsets of this segment. This is repeated until a minimum value, $Q_{\min }$, of the least squares functional is obtained. 
The direct search was carried out according to the strategy described above. Map combinations involving 3 and 4 terms were tried, i.e., $K=3$ and $K=4$ in Eq. (7). It was found that a combination of four maps produced $Q$ values at least an order of magnitude smaller than the fits with three maps. Hence only the four map combination has been used in the present study to obtain all the results discussed below. The map parameters corresponding to the best case for each location in the flame are given in Table 2. It can be seen from this table that the four bifurcation parameters, $b_{k}$, of the map combination, do not change with fits to different locations in the flame. The iteration frequencies, $\omega_{k}$, also do not change; but some slight changes are observed in the duration counts, $d_{k}$. Most of the changes occur in the amplitude factors, $\alpha_{k}$. From our experience in this research, the $b_{k}$ had the strongest influence on $Q_{\text {min }}$. But, the particular combination of $b_{k}$ shown in Table 2 turns out to be surprisingly stable with respect to data fits at different flame locations.

The implicitness factor, $\theta$, was not a part of the direct search but was manually modified as the search progressed, and its optimal value is also shown in Table 2. From Eq. (8) it can be seen that larger values of $\theta$, imply less "memory" of a time series of its past behavior. The monotonically increasing trend of $\theta$ (from Table $2(\mathrm{a}-\mathrm{c})$ ), indicates that as we go higher in the flame, the prevailing turbulent field exerts a greater influence on the fluctuations of soot volume fraction; and consequently, there is a progressive weakening of the influence of past history.

The search procedure produced $Q_{\min } \sim \mathcal{O}\left(10^{-6}\right)$ for all three locations in the flame considered here. To put this into context, we note that the maximum value was $Q_{\max } \sim$ $\mathcal{O}\left(10^{-2}\right)$. The time series for the best cases are shown in Fig. 2(d-f); the corresponding power spectra and delay maps (delay $=4$ ) are shown in Fig. 3(d-f) and 4(d-f), respectively, and the histograms of the intermittency distributions are presented in Figure 5(d-f). These results have to be compared with the experimental data, Figs. 2(a-c), 3(a-c), 4(a-c) and $5(\mathrm{a}-\mathrm{c})$, respectively. The quantitative values for the various statistical properties discussed earlier are presented in Table 1 for both the modeled and experimental data at the $h=4.0$ $\mathrm{cm}$. location. Similar tables can be constructed for the other locations but are not presented here. 
From the experience gained during this research, it appeared that the autocorrelations were not important in obtaining a good match between modeled and computed data in the present case; hence, these were ultimately removed from the analysis. The skewness and flatness properties also produced only a minor contribution to the fitting process. However, it was found that the intermittency factors were critical for a good data fit. The normalized weights shown in the fifth column of Table 1 reflect this, with smaller weights assigned to skewness and flatness while the intermittency factors are heavily weighted. The algorithm used to specify the weights was designed such that every important property had a balanced contribution to $Q$. In particular, the raw weights, $\overline{\phi_{i}}$, were specified as

$$
\overline{\phi_{i}}= \begin{cases}1.0 & \text { for } i=1, \ldots, 21, \\ 0.1 & \text { for } i=22,23 \text { (skewness \& flatness) } \\ \frac{1}{\left(p_{i}^{\text {meas }}\right)^{2}} & \text { for } i=24, \ldots, 33 \text { (intermittencies) }\end{cases}
$$

and the normalized weights, $\phi_{i}$, were computed from these by

$$
\phi_{i}=\frac{\overline{\phi_{i}}}{\sum_{i=1}^{N_{p}} \overline{\phi_{i}}} .
$$

The time series, (Figs. 2(a-c) \& 2(d-f)), are in reasonably good qualitative agreement in the sense that corresponding figures have similar qualitative appearance. (One would not expect pointwise detailed agreement for turbulent behavior.) From these figures it can be seen that the time averages and the amplitudes and frequency of oscillations match very well and follow the correct trends along the flame axis. The different scales of "structures" are replicated in detail in the modeled time series. The distinct amplitudes $\alpha_{k}$ in Table 2 give quantitative information about the relative sizes of the different scales. There is generally good quantitative agreement too, as can be seen from Table 1.

The agreement in the power spectra, Fig. 3, is quite striking particularly because these were not part of the modeling process. Apart from the fairly good agreement in the overall power levels and decay rates, it can be seen that the model is able to replicate the subharmonic bifurcation indicated by the $6 \mathrm{~Hz}$ and $12 \mathrm{~Hz}$ peaks. The experimental data show a sharp peak at $10.8 \mathrm{~Hz}$ (probably due to quasi-periodicity); the model is able to replicate this at the correct power but at a frequency of $10 \mathrm{~Hz}$. This may be an inherent limitation of 
the logistic map in producing quasi-periodic bifurcations. The delay maps in Fig. 4, appear to be topologically equivalent. The delay map for the modeled time series at $h=7.0 \mathrm{~cm}$. location, Fig. 4(f), has at least two points where the trajectories are clustered together which is indicative of an underlying periodic behavior. A hint of this behavior can also be seen in the corresponding experimental delay map, Fig 4(c), where we can see trajectories loosely clustered into two separate lobes.

The skewness in the experimental intermittency distribution, Fig. 5(a-c), changes along the flame. The same general behavior is also observed in the intermittency distribution of the model, Fig. 5(d-f). But there are some differences in the details, e.g., the intermittency distribution in the modeled case shown in Fig. 5(d) is more symmetric than the distribution in the corresponding experimental case shown in Fig. 5(a). One probable cause for this is the weights associated with $u_{\min }$ and $u_{\max }$. These properties are a part of the fitting procedure and influence the intermittency calculations because they determine the end points, $g_{0}$ and $g_{M}$, in Eq. (14). If these properties are not matched closely between experimental and modeled data then this would imply that the intermittency calculations are being done in

slightly different intervals in the two cases, leading to the differences observed in Figs. 5(a) and $5(\mathrm{~d})$. The remedy to this is to match $u_{\min }$ and $u_{\max }$ very closely for experimental and modeled data by using greater weights on these properties. This will be implemented in future research involving the present data fitting approach.

\subsection{Predictions from Maps with Interpolated Parameters}

As discussed earlier, here we will attempt to predict the time series and their quantifying measures at intermediate locations in the flame with the map parameters obtained by interpolation of the parameters calculated by data-fitting in the previous section. Experimental time series at two intermediate locations $(h=2.0 \mathrm{~cm}$. and $h=6.0 \mathrm{~cm}$.) are available and are shown in Figs. 6(a,b). These will be used to compare the predictions from the interpolated maps.

We use a quadratic polynomial interpolation to obtain the parameters of the map combination at the intermediate locations. It should be noted from Table 2 that some parameters 
which are constant for all the locations (including all the $b_{k}$ values) are not interpolated. Interpolation is done for all the amplitudes, $\alpha_{k}$, and the duration count, $d_{1}$, that change with location. It can be seen from Table 2 that parameters like $\alpha_{1}$ and $\alpha_{3}$ are monotonically increasing (or decreasing), and reasonable results can be expected by interpolation of these parameters. Other parameters like $\alpha_{2}$ and $\alpha_{4}$ vary quite nonlinearly (maybe chaotically) and it is not clear what a good interpolating function for these ought to be. Here we use quadratic interpolation for simplicity and for the lack of any physical reasons for doing otherwise. The interpolated set of map parameters are shown in Table 3 for $h=2.0 \mathrm{~cm}$. and $h=6.0 \mathrm{~cm}$. locations, respectively. The map time series obtained for these parameters are shown in Figs. 6(c,d). As was done before, we generate psds and intermittency histograms for both the experimental data (shown in Figs. $7(a, b)$ and $8(a, b)$ ) and also for data from the interpolated maps (shown in Figs. $7(\mathrm{c}, \mathrm{d})$ and $8(\mathrm{c}, \mathrm{d})$ ). We compute the statistical properties and the least-squares functional, $Q$, which are shown in Table 4 for only the $h=2.0 \mathrm{~cm}$. axial location.

Despite the fact that we are attempting the difficult task of trying to predict the behavior of a higly nonlinear system, the experimental data, with another simpler nonlinear system, the chaotic map combination, the qualitative agreement in the time series, Figs. $6(\mathrm{a}, \mathrm{b})$ and $6(c, d)$, is fairly good in terms of mean values, amplitudes and oscillation frequencies. It can be seen from Table 4 that although the quantitative agreement between predicted and measured statistics is good, the functional, $Q$, obtained from the maps with the interpolated parameters is an order of magnitude larger than that obtained by data-fitted maps. This is to be expected because the quadratic fit is only a convenient approximation that is used here to interpolate map parameters some of which vary in a nonlinear fashion for different locations on the flame. It can be seen that the power spectra, Figs. $7(\mathrm{a}, \mathrm{b})$ and $7(\mathrm{c}, \mathrm{d})$, also agree well not only in terms of the gross features like overall power levels and decay rates, but also in the finer details of the subharmonic and quasiperiodic peaks. The overall match in itermittency factors, Figs. $8(a, b)$ and $8(c, d)$, is not very good although they roughly show the correct skewness in oscillatory data. The peaks in the intermittency data from the experimental time series are not reproduced in the predicted data from the chaotic map models. This only emphasizes our previous observation from the data-fitting exercise that 
the intermittency factors are important in obtaining a good match between the two time series. A close match between intermittency factors cannot be guaranteed without resorting to a data-fitting procedure.

\section{SUMMARY AND CONCLUSIONS}

In this paper we have discussed a novel approach to investigate the radiation-turbulence interactions in diffusion flames. We have modeled experimental data from a soot-laden turbulent diffusion flame produced with a co-flow burner. The data show strong evidence of a subharmonic bifurcation sequence from periodic behavior at the tip of the burner to fully turbulent at some height (depending on air/fuel ratio) above the burner. On the basis of these observations we have constructed chaotic map models of this behavior using linear combinations of a modified logistic map.

We can draw several conclusions from this study that we hope may lead to innovations in turbulence modeling approaches:

- The radiation-turbulence interaction phenomenon in a soot-laden flame is evidently deterministic, rather than random, as has been reported earlier [1].

- It is possible to devise fairly simple algebraic models of this quite complicated flow situation, but unlike earlier attempts with other physical systems, our model displays correct bifurcation sequences in addition to accurate behavior at individual parameter settings within the sequence. This implies a potential for "universality" and indicates that such models should be extremely useful in the context of the ATD/chaotic map turbulence modeling formalism as well as possibly in LES.

\section{Acknowledgements}

This work was supported by the DOE-PETC Advanced University Coal Research Program Grant No: DE-FG22-93PC93210. Ms. Chung's work was supported under a NSF Research Experiences for Undergraduates (REU) Fellowship at the University of Kentucky. 


\section{References}

[1] Mengü̧, M. P., McDonough, J. M., Manickavasagam, S., Mukerji, S., Swabb, S. and Ghosal, S., Fluctuations of soot in turbulent diffusion flames: Experimental data and chaotic map models. Presented at ASME International Mechanical Engineering Congress and Exposition, Atlanta, GA, Nov. 17-22, 1996.

[2] Faeth, G. M., Jeng, S. M. and Gore, J., Radiation from flames. In Heat Transfer in Fire and Combustion Systems, HTD-Vol. 45, ed. C. K. Law et al., ASME, New York, 1985 , pp. 137-151.

[3] Grosshandler, W. L. and Joulain, P., The effect of large scale fluctuations on flame radiation. In Progress in Astronautics and Aeronautics, Vol. 105, Part II. AIAA, Washington, 1986, pp. 123-152.

[4] Gore, J. P., Jeng, S. M. and Faeth, G. M., Spectral and total radiation properties of turbulent hydrogen/air diffusion flames. ASME Journal of Heat Transfer, 1988, 110, $173-181$.

[5] Faeth, G. M., Gore, J. P., Chuech, S. G. and Jeng, S. M., Radiation from turbulent diffusion flames. In Annual Review of Numerical Fluid Mechanics and Heat Transfer, ed. C. L. Tien and T. C. Chawla. Hemisphere, New York, 1989, pp. 1-38.

[6] Kounalakis, M. E., Sivathanu, Y. R. and Faeth, G. M., Infrared radiation statistics of nonluminous turbulent diffusion flames. ASME Journal of Heat Transfer, 1991, 113, $437-445$.

[7] Sivathanu, Y. R. and Gore, J. P., Coupled radiation and soot kinetics calculations in laminar acetylene/air diffusion flames. Combustion and Flame, 1994, 97, 161-172.

[8] Freitas, C. J., Perspective: Selected benchmarks from commercial CFD codes. Journal of Fluids Engineering, 1995, 117, 208-218.

[9] McDonough, J. M., On intrinsic errors in turbulence models based on Reynolds-averaged Navier-Stokes equations. Fluid Mechanics Research, 1995, 22, 1-29. 
[10] Hylin, E. C. and McDonough, J. M., Theoretical development of a stochastic model for small-scale turbulence in an additive decomposition of the Navier-Stokes equations. Mechanical Engineering Report CFD-02-96, University of Kentucky, Lexington, KY, 1996.

[11] Sreenivisan, K. R. and Ramshankar, R., Transition intermittency in open flows, and intermittency routes to chaos. Physica, 1986, 23D, 246-258.

[12] McDonough, J. M., Mukerji, S. and Chung, S., A data-fitting procedure for chaotic time series. To appear in Applied Mathematics and Computation, 1998.

[13] Collet, P. and Eckmann, J.-P., Iterated Maps on the Interval as Dynamical Systems, Birkhäuser-Boston, Boston, 1980.

[14] Feigenbaum, M. J., Quantitative universality for a class of nonlinear transformations. Journal of Statistical Physics, 1978, 19, 25-52.

[15] Pulliam, T. H. and Vastano, J. A., Transition to chaos in an open unforced 2D flow. Journal of Computational Physics, 1993, 105, 133-149.

[16] Frisch, U., Turbulence, Cambridge University Press, Cambridge, 1995.

[17] McDonough, J. M., Bywater, R. J. and Buell, J. C., An investigation of strange attractor theory and small-scale turbulence. AIAA Paper 84-1674, 1984.

[18] McDonough, J. M., Buell, J. C. and Bywater, R. J., A comparison of routes to a strange attractor in one-dimensional local models of turbulent free and forced convection. ASME Paper 84-WA/HT-16, 1984.

[19] McDonough, J. M. and Bywater, R. J., Large-scale effects on local small-scale chaotic solutions to Burgers' equation. AIAA Journal, 1986, 24, 1924-1930.

[20] McDonough, J. M. and Bywater, R. J., Turbulent solutions from an unaveraged, additive decomposition of Burgers' equation. In Forum on Turbulent Flows, ed. W. W. Bower and M. J. Morris. American Society of Mechanical Engineers (ASME), 1989, pp. 7-12. 
[21] McDonough, J. M. and Wang, D., Additive turbulent decomposition: A highly parallelizable turbulence simulation technique. In Parallel Computational Fluid Dynamics: New Algorithms and Applications, ed. N. Satofuka et al.. Elsevier Science B.V. Amsterdam, 1995, pp. 129-136.

[22] Mukerji, S. and McDonough, J. M., Parallel computation of 3-D small-scale turbulence via additive turbulent decomposition. In Parallel Computational Fluid Dynamics: Implementations and Results Using Parallel Computers, ed. A. Acer et al.. Elsevier Science B.V. Amsterdam, 1996, pp. $465-472$.

[23] McDonough, J. M., Yang, Y. and Hylin, E. C., Modeling time-dependent turbulent flow over a backward-facing step via additive turbulent decomposition and chaotic algebraic maps. In Proceedings of the First Asian Computational Fluid Dynamics Conference, ed. W. H. Hui, Y.-K. Kwok, and J. R. Chasnov. 1995, pp. 747-752.

[24] McDonough, J. M., Wang, D. and Mengüç, M. P., Radiation-turbulence interaction in flames using additive turbulent decomposition. In Radiative Transfer - I : Proceedings of the First International Symposium on Radiative Transfer, ed. M. P. Mengüç. Begell House, New York, 1996.

[25] Salazar, A. J., McDonough, J. M. and Mukerji, S., Transient heat transfer and fluid flow over an internal turbulence promoter via ATD/chaotic-map modeling. ASME Paper FEDSM97-3166, 1997.

[26] Marion, M. and Temam, R., Nonlinear Galerkin methods. SIAM Journal on Numerical Analysis, 1989, 26, 1139-1157.

[27] Brown, R. M., Perry, P. and Shen, Z., The additive turbulent decomposition for the twodimensional incompressible Navier-Stokes equations: convergence theorems and error estimates. To appear in SIAM Journal of Mathematical Analysis, 1998.

[28] Wilcox, D. C., Turbulence Modeling for CFD, DCW Industries, Inc., La Cañada, CA, 1993. 
[29] Tennekes, H. and Lumley, J. L., A First Course in Turbulence, MIT Press, Cambridge MA, 1973.

[30] Buckmaster, J. and Peters, N., The infinite candle and its stability - A paradigm for flickering turbelent diffusion flames. Proceedings of the 21st International Symposium on Combustion, 1986, The Combustion Institute, Pittsburgh, PA, pp. 1829-1936.

[31] Ruelle, D. and Takens, F., On the nature of turbulence. In Communications in Mathematical Physics, 1971, 20, pp. 167-192.

[32] Guckenheimer, J. and Holmes, P., Nonlinear Oscillations, Dynamical Systems, and Bifurcations of Vector Fields, Springer Verlag, New York, 1983.

[33] Bergé, P., Pomeau, Y. and Vidal, C., Order within Chaos, John Wiley and Sons, New York, 1984. 


\begin{tabular}{|c|c|c|c|c|c|}
\hline $\begin{array}{c}\text { Property } \\
\text { No. } \\
i\end{array}$ & Description & $\begin{array}{c}\text { Measured } \\
\text { Value } \\
p_{i}^{\text {meas }} \\
\end{array}$ & $\begin{array}{c}\text { Modeled } \\
\text { Value } \\
p_{i}^{\text {model }} \\
\end{array}$ & $\begin{array}{c}\text { Weights } \\
\phi_{i} \\
\end{array}$ & $\begin{array}{c}\text { Contribution } \\
\text { To } Q \\
\phi_{i}\left(\delta p_{i}\right)^{2}\end{array}$ \\
\hline 1 & Average $(\bar{u})$ & $-2.205[-3]$ & $-3.022[-1]$ & $2.296[-6]$ & $2.066[-7]$ \\
\hline 2 & Positive average $(\bar{u})$ & $2.620[0]$ & $2.562[0]$ & $2.296[-6]$ & $7.691[-9]$ \\
\hline 3 & Negative average $(\bar{u})$ & $-2.608[0]$ & $-2.863[0]$ & $2.296[-6]$ & $1.493[-7]$ \\
\hline 4 & Average variation $(\bar{V}(u))$ & $3.132[0]$ & $3.052[0]$ & $2.296[-6]$ & $1.458[-8]$ \\
\hline 5 & Positive average variation $\left(\bar{V}^{+}(u)\right)$ & $2.553[0]$ & $2.099[0]$ & $2.296[-6]$ & $4.729[-7]$ \\
\hline 6 & Negative average variation $\left(\bar{V}^{-}(u)\right)$ & $2.367[0]$ & $2.285[0]$ & $2.296[-6]$ & $1.574[-8]$ \\
\hline 7 & Slope sign changes, total $\left(f_{s \pm}\right)$ & $7.756[-1]$ & $7.936[-1]$ & $2.296[-6]$ & $7.440[-10]$ \\
\hline 8 & Slope sign changes, $u$ positive $\left(f_{s^{ \pm}}^{+}\right)$ & $7.909[-1]$ & $7.849[-1]$ & $2.296[-6]$ & $8.200[-11]$ \\
\hline 9 & Slope sign changes, $u$ negative $\left(f_{s^{ \pm}}^{ \pm}\right)$ & $7.603[-1]$ & $8.013[-1]$ & $2.296[-6]$ & $3.853[-9]$ \\
\hline 10 & Time average $L^{i}$ norm & $2.614[0]$ & $2.721[0]$ & $2.296[-6]$ & $2.628[-8]$ \\
\hline 11 & Time average $L^{2}$ norm & $1.833[-1]$ & $1.831[-1]$ & $2.296[-6]$ & $1.792[-13]$ \\
\hline 12 & Overall minimum $\left(u_{\min }\right)$ & $-1.547[1]$ & $-1.549[1]$ & $2.296[-6]$ & $4.673[-10]$ \\
\hline 13 & Overall maximum $\left(u_{\max }\right)$ & $1.262[1]$ & $1.260[1]$ & $2.296[-6]$ & $1.513[-9]$ \\
\hline 14 & Positive minimum $\left(u_{m i n}^{+}\right)$ & $1.118[-3]$ & $5.414[-4]$ & $2.296[-6]$ & $7.628[-13]$ \\
\hline 15 & Positive maximum $\left(u_{\max }^{+}\right)$ & $1.262[1]$ & $1.260[1]$ & $2.296[-6]$ & $1.513[-9]^{2}$ \\
\hline 16 & Negative minimum $\left(u_{m i n}^{-}\right)$ & $-1.547[1]$ & $-1.549[1]$ & $2.296[-6]$ & $4.673[-10]$ \\
\hline 17 & Negative maximum $\left(u_{m a x}^{-}\right)$ & $0.000[0]$ & $0.000[0]$ & $2.296[-6]$ & $0.000[0]$ \\
\hline 18 & Number of zero crossings/time $\left(N_{0} / N\right)$ & $4.158[-1]$ & $3.681[-1]$ & $2.296[-6]$ & $5.225[-9]$ \\
\hline 19 & Number of $\bar{u}$ crossings/time $\left(N_{0} / N\right)$ & $4.164[-1]$ & $3.671[-1]$ & $2.296[-6]$ & $5.581[-9]$ \\
\hline 20 & Number of $\bar{u}$ crossings/time $\left(N_{\text {mean }}+/ N\right)$ & $2.920[-1]$ & $2.810[-1]$ & $2.296[-6]$ & $2.779[-10]$ \\
\hline 21 & Number of $\bar{u}$ crossings/time $\left(N_{\text {mean }}-/ N\right)$ & $2.703[-1]$ & $2.961[-1]$ & $2.296[-6]$ & $1.529[-9]$ \\
\hline 22 & Skewness & $-1.621[-1]$ & $-3.558[-1]$ & $2.296[-7]$ & $8.612[-9]$ \\
\hline 23 & Flatness & $3.811[0]$ & $2.889[0]$ & $2.296[-7]$ & $1.955[-7]$ \\
\hline 24 & Intermittency factor $\left(f_{I_{1}}\right)$ & & & $5.203[-1]$ & $1.504[-6]$ \\
\hline 25 & Intermittency factor $\left(f_{I_{2}}\right)$ & & & $1.434[-1]$ & $2.425[-7]$ \\
\hline 26 & Intermittency factor $\left(f_{r_{3}}\right)$ & Inter- & Inter- & $1.122[-2]$ & $2.874[-8]$ \\
\hline 27 & Intermittency factor $\left(f_{I_{4}}\right)$ & mittency & mittency & $4.656[-4]$ & $5.937[-7]$ \\
\hline 28 & Intermittency factor $\left(f_{I_{5}}\right)$ & factors & factors & $4.410[-5]$ & $7.059[-12]$ \\
\hline 29 & Intermittency factor $\left(f_{I_{6}}\right)$ & shown & shown & $2.075[-5]$ & $4.985[-8]$ \\
\hline 30 & Intermittency factor $\left(f_{I_{7}}\right)$ & graphically & graphically & $4.326[-5]$ & $2.244[-9]$ \\
\hline 31 & Intermittency factor $\left(f_{I_{s}}\right)$ & in Fig. $5(\mathrm{~b})$ & in Fig. $5(e)$ & $3.649[-4]$ & $3.650[-10]$ \\
\hline 32 & Intermittency factor $\left(f_{I_{9}}\right)$ & & & $9.309[-3]$ & $8.583[-7]$ \\
\hline 33 & Intermittency factor $\left(f_{I_{10}}\right)$ & & & $3.148[-1]$ & $6.1 \div 2[-7]$ \\
\hline
\end{tabular}

Least-squares functional, $Q=5.016[-6]$.

Maximum contribution to $Q$ is from property $24, f_{I_{1}}$.

Table 1: Statistical properties of data from experiments and best fit chaotic map model for location $h=4.0 \mathrm{~cm}$ in the flame. 


\begin{tabular}{|c|c|c|c|c|}
\hline Map, $k$ & $\alpha_{k}$ & $b_{k}$ & $\omega_{k}$ & $d_{k}$ \\
\hline 1 & $2.350[0]$ & -3.82260 & 2000 & 200 \\
2 & $1.500[0]$ & -3.93864 & 170 & 158 \\
3 & $-6.310[-1]$ & -4.00956 & 1 & 1 \\
4 & $2.000[-1]$ & -4.13440 & 1 & 1 \\
\hline
\end{tabular}

$\theta=0.110$

(a)

\begin{tabular}{|c|c|c|c|c|}
\hline Map, $k$ & $\alpha_{k}$ & $b_{k}$ & $\omega_{k}$ & $d_{k}$ \\
\hline 1 & $2.282[1]$ & -3.82260 & 2000 & 90 \\
2 & $9.050[0]$ & -3.93864 & 170 & 158 \\
3 & $-1.700[1]$ & -4.00956 & 1 & 1 \\
4 & $-1.800[0]$ & -4.13440 & 1 & 1 \\
\hline
\end{tabular}

$\theta=0.277$

(b)

\begin{tabular}{|c|c|c|c|c|}
\hline Map, $k$ & $\alpha_{k}$ & $b_{k}$ & $\omega_{k}$ & $d_{k}$ \\
\hline 1 & $4.198[1]$ & -3.82260 & 2000 & 90 \\
2 & $2.900[0]$ & -3.93864 & 170 & 158 \\
3 & $-4.420[1]$ & -4.00956 & 1 & 1 \\
4 & $2.000[0]$ & -4.13440 & 1 & 1 \\
\hline
\end{tabular}

$\theta=0.320$

(c)

Table 2: Map parameters corresponding to the best data fits for different locations along the flame. (a) $h=0.5 \mathrm{~cm}$, (b) $h=4.0 \mathrm{~cm}$ and (c) $h=7.0 \mathrm{~cm}$. 


\begin{tabular}{|c|c|c|c|c|}
\hline Map, $k$ & $\alpha_{k}$ & $b_{k}$ & $\omega_{k}$ & $d_{k}$ \\
\hline 1 & $1.087[1]$ & -3.82260 & 2000 & 138 \\
2 & $6.677[0]$ & -3.93864 & 170 & 158 \\
3 & $-5.620[0]$ & -4.00956 & 1 & 1 \\
4 & $-1.505[0]$ & -4.13440 & 1 & 1 \\
\hline
\end{tabular}

$\theta=0.197$

(a)

\begin{tabular}{|c|c|c|c|c|}
\hline Map, $k$ & $\alpha_{k}$ & $b_{k}$ & $\omega_{k}$ & $d_{k}$ \\
\hline 1 & $3.543[1]$ & -3.82260 & 2000 & 80 \\
2 & $6.245[0]$ & -3.93864 & 170 & 158 \\
3 & $-3.378[1]$ & -4.00956 & 1 & 1 \\
4 & $1.719[0]$ & -4.13440 & 1 & 1 \\
\hline
\end{tabular}

$\theta=0.316$

(b)

Table 3: Map parameters obtained by quadratic interpolation of those of Table 2 for intermediate locations along the flame. (a) $h=2.0 \mathrm{~cm}$ and (b) $h=6.0 \mathrm{~cm}$. 


\begin{tabular}{|c|c|c|c|c|c|}
\hline $\begin{array}{c}\text { Property } \\
\text { No. } \\
i \\
\end{array}$ & Description & $\begin{array}{c}\text { Measured } \\
\text { Value } \\
p_{i}^{\text {meas }} \\
\end{array}$ & $\begin{array}{c}\text { Modeled } \\
\text { Value } \\
p_{i}^{\text {model }} \\
\end{array}$ & $\begin{array}{c}\text { Weights } \\
\phi_{i}\end{array}$ & $\begin{array}{c}\text { Contribution } \\
\text { To } Q \\
\phi_{i}\left(\delta p_{i}\right)^{2}\end{array}$ \\
\hline 1 & Average $(\bar{u})$ & $-8.731[-4]$ & $-3.923[-1]$ & $8.756[-7]$ & $1.341[-7]$ \\
\hline 2 & Positive average $\left(\overline{u^{+}}\right)$ & $6.243[-1]$ & $8.149[-1]$ & $8.756[-7]$ & $3.182[-8]$ \\
\hline 3 & Negative average $(\bar{u})$ & $-6.811[-1]$ & $-1.150[0]$ & $8.756[-7]$ & $1.929[-7]$ \\
\hline 4 & Average variation $(\bar{V}(u))$ & $7.208[-1]$ & $1.059[0]$ & $8.756[-7]$ & $1.002[-7]$ \\
\hline 5 & Positive average variation $\left(\bar{V}^{+}(u)\right)$ & $5.927[-1]$ & $7.117[-1]$ & $8.756[-7]$ & $1.239[-8]$ \\
\hline 6 & Negative average variation $\left(\bar{V}^{-}(u)\right)$ & $6.097[-1]$ & $9.428[-1]$ & $8.756[-7]$ & $9.717[-8]$ \\
\hline 7 & Slope sign changes, total $\left(f_{s \pm}\right)$ & $7.747[-1]$ & $7.810[-1]$ & $8.756[-7]$ & $3.477[-11]$ \\
\hline 8 & Slope sign changes, $u$ positive $\left(f_{s \pm}^{+}\right)$ & $7.685[-1]$ & $7.848[-1]$ & $8.756[-7]$ & $2.322[-10]$ \\
\hline 9 & Slope sign changes, $u$ negative $\left(f_{s \pm}^{-}\right)$ & $7.813[-1]$ & $7.785[-1]$ & $8.756[-7]$ & $6.819[-12]$ \\
\hline 10 & Time average $L^{1}$ norm & $6.514[-1]$ & $1.021[0]$ & $8.756[-7]$ & $1.195[-7]$ \\
\hline 11 & Time average $L^{2}$ norm & $4.609[-2]$ & $7.062[-2]$ & $8.756[-7]$ & $5.270[-10]$ \\
\hline 12 & Overall minimum $\left(u_{\min }\right)$ & $-4.741[0]$ & $-5.283[0]$ & $8.756[-7]$ & $2.569[-7]$ \\
\hline 13 & Overall maximum $\left(u_{\max }\right)$ & $2.587[0]$ & $3.031[0]$ & $8.756[-7]$ & $1.726[-7]$ \\
\hline 14 & Positive minimum $\left(u_{\min }^{+}\right)$ & $3.034[-4]$ & $1.211[-3]$ & $8.756[-7]$ & $7.219[-13]$ \\
\hline 15 & Positive maximum $\left(u_{\max }^{+}\right)$ & $2.587[0]$ & $3.031[0]$ & $8.756[-7]$ & $1.726[-7]$ \\
\hline 16 & Negative minimum $\left(u_{\min }^{-}\right)$ & $-4.741[0]$ & $-5.283[0]$ & $8.756[-7]$ & $2.569[-7]$ \\
\hline 17 & Negative maximum $\left(u_{m a x}^{-}\right)$ & $0.000[0]$ & $0.000[0]$ & $8.756[-7]$ & $0.000[0]$ \\
\hline 18 & Number of zero crossings/time ( & $3.801[-1]$ & $3.259[-1]$ & $8.756[-7]$ & $2.573[-9]$ \\
\hline 19 & Number of $\bar{u}$ crossings/time $\left(N_{0} / N\right)$ & $3.801[-1]$ & $3.481[-1]$ & $8.756[-7]$ & $8.970[-10]$ \\
\hline 20 & Number of $\bar{u}$ crossings/time $\left(N_{\text {mean }}+/ N\right)$ & $3.065[-1]$ & $2.223[-1]$ & $8.756[-7]$ & $6.195[-9]$ \\
\hline 21 & Number of $\bar{u}$ crossings/time $\left(N_{\text {mean }}-/ N\right)$ & $2.124[-1]$ & $2.897[-1]$ & $8.756[-7]$ & $5.220[-9]$ \\
\hline 22 & Skewness & $-5.625[-1]$ & $-1.097[0]$ & $8.756[-8]$ & $2.506[-8]$ \\
\hline 23 & Flatness & $4.414[0]$ & $3.362[0]$ & $8.756[-8]$ & $9.677[-8]$ \\
\hline 24 & Intermittency fact & & & $7.233[-1]$ & $2.345[-6]$ \\
\hline 25 & Intermittency factor $\left(f_{I_{2}}\right)$ & & & $1.985[-1]$ & $3.177[-6]$ \\
\hline 26 & Intermittency factor $\left(f_{I_{3}}\right)$ & Inter- & Inter- & $4.734[-2]$ & $1.227[-5]$ \\
\hline 27 & Intermittency factor $\left(f_{I_{4}}\right)$ & mittency & mittency & $3.506[-3]$ & $9.890[-6]$ \\
\hline 28 & Intermittency factor $\left(f_{I_{5}}\right)$ & factors & factors & $1.822[-4]$ & $1.112[-6]$ \\
\hline 29 & Intermittency factor $\left(f_{I_{B}}\right)$ & shown & shown & $1.886[-5]$ & $4.303[-9]$ \\
\hline 30 & Intermittency factor $\left(f_{I_{7}}\right)$ & graphically & graphically & $7.096[-6]$ & $1.269[-7]$ \\
\hline 31 & Intermittency factor $\left(f_{I_{8}}\right)$ & in Fig. 8(a) & in Fig. $8(\mathrm{c})$ & $1.498[-5]$ & $1.445[-7]$ \\
\hline 32 & Intermittency factor $\left(f_{I_{g}}\right)$ & & & $1.722[-4]$ & $2.564[-8]$ \\
\hline 33 & Intermittency factor $\left(f_{I_{10}}\right)$ & & & $2.694[-2]$ & $2.760[-7]$ \\
\hline
\end{tabular}

Least-squares functional, $Q=3.106[-5]$.

Maximum contribution to $Q$ is from property $26, f_{I_{3}}$.

Table 4: Statistical properties of data from experiments and predictions from chaotic map model with interpolated parameters for location $h=2.0 \mathrm{~cm}$ in the flame. 


\section{LIST OF FIGURES}

1. Photograph of the ethylene diffusion flame at four successive time slices.

2. Time series of scattered light intensity from soot particles at different axial locations along the flame. (a) $h=0.5 \mathrm{~cm}$, (b) $h=4.0 \mathrm{~cm}$ and (c) $h=7.0 \mathrm{~cm}$. Corresponding time series obtained from the best fits of linear combination of logistic maps for locations (d) $h=0.5 \mathrm{~cm}$, (e) $h=4.0 \mathrm{~cm}$ and (f) $h=7.0 \mathrm{~cm}$.

3. Power spectra from experimental time series of soot scattering intensity at different locations. (a) $h=0.5 \mathrm{~cm}$, (b) $h=4.0 \mathrm{~cm}$ and (c) $h=7.0 \mathrm{~cm}$. Power spectra of time series obtained from the best fit combination of logistic maps (d) $h=0.5 \mathrm{~cm}$, (e) $h=4.0 \mathrm{~cm}$ and (f) $h=7.0 \mathrm{~cm}$.

4. Delay maps (with delay=4) of the experimental time series of Fig. 2 at different axial locations along the flame. (a) $h=0.5 \mathrm{~cm}$, (b) $h=4.0 \mathrm{~cm}$ and (c) $h=7.0 \mathrm{~cm}$. Delay maps (with delay $=4$ ) of the time series obtained from the best fit combination of logistic maps. (d) $h=0.5 \mathrm{~cm}$, (e) $h=4.0 \mathrm{~cm}$ and (f) $h=7.0 \mathrm{~cm}$.

5. Intermittency distributions of the experimental time series at different axial locations along the flame. (a) $h=0.5 \mathrm{~cm}$, (b) $h=4.0 \mathrm{~cm}$ and (c) $h=7.0 \mathrm{~cm}$. Intermittency distributions of the time series obtained from the best fit combination of logistic maps. (d) $h=0.5 \mathrm{~cm}$, (e) $h=4.0 \mathrm{~cm}$ and (f) $h=7.0 \mathrm{~cm}$.

6. Experimental time series of light scattering intensities at two intermediate locations along the flame. (a) $h=2.0 \mathrm{~cm}$ and (b) $h=6.0 \mathrm{~cm}$. Corresponding predicted time series obtained from linear combination of logistic maps with interpolated parameters. (c) $h=2.0 \mathrm{~cm}$ and (d) $h=6.0 \mathrm{~cm}$.

7. Power spectra from experimental time series of soot scattering intensity at intermediate locations (a) $h=2.0 \mathrm{~cm}$ and (b) $h=6.0 \mathrm{~cm}$, along the flame. Corresponding power spectra of time series obtained from a combination of logistic maps with interpolated parameters. (c) $h=2.0 \mathrm{~cm}$ and (d) $h=6.0 \mathrm{~cm}$.

8. Intermittency distributions of the experimental time series at intermediate locations (a) $h=2.0 \mathrm{~cm}$ and (b) $h=6.0 \mathrm{~cm}$, along the flame. Intermittency distributions of the time series obtained from a combination of interpolated logistic maps. (c) $h=2.0 \mathrm{~cm}$ and (d) $h=6.0 \mathrm{~cm}$. 


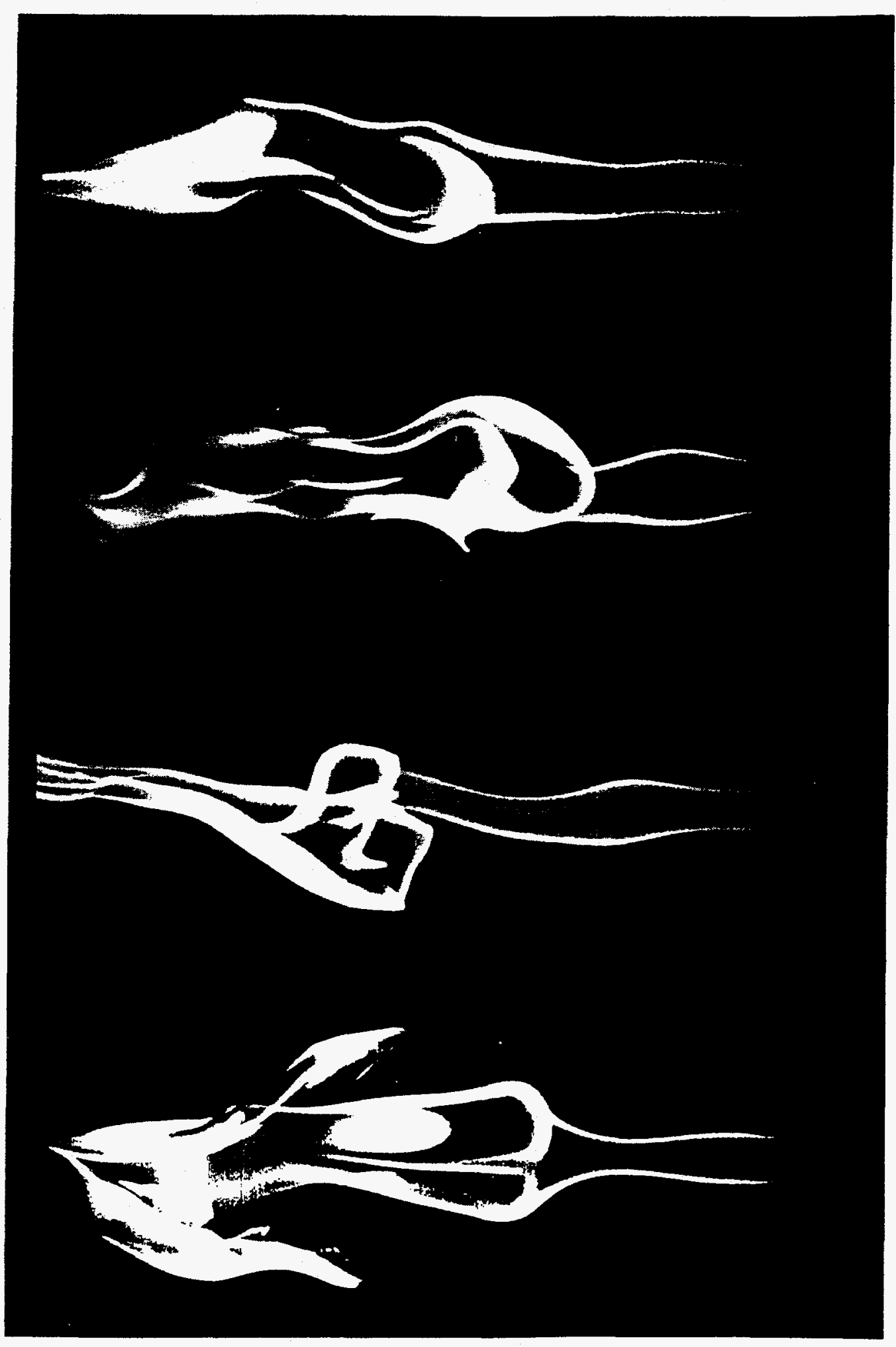



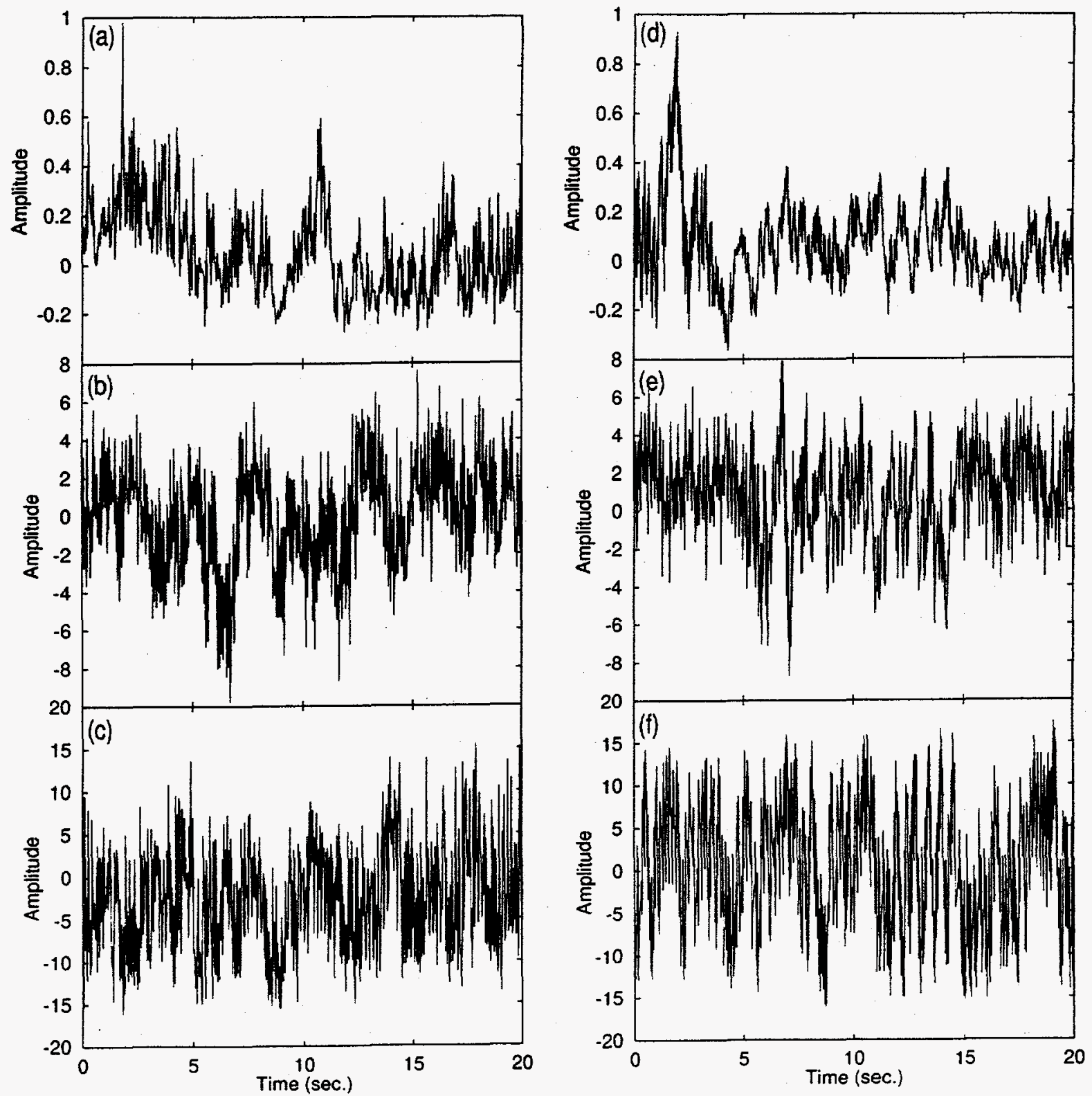

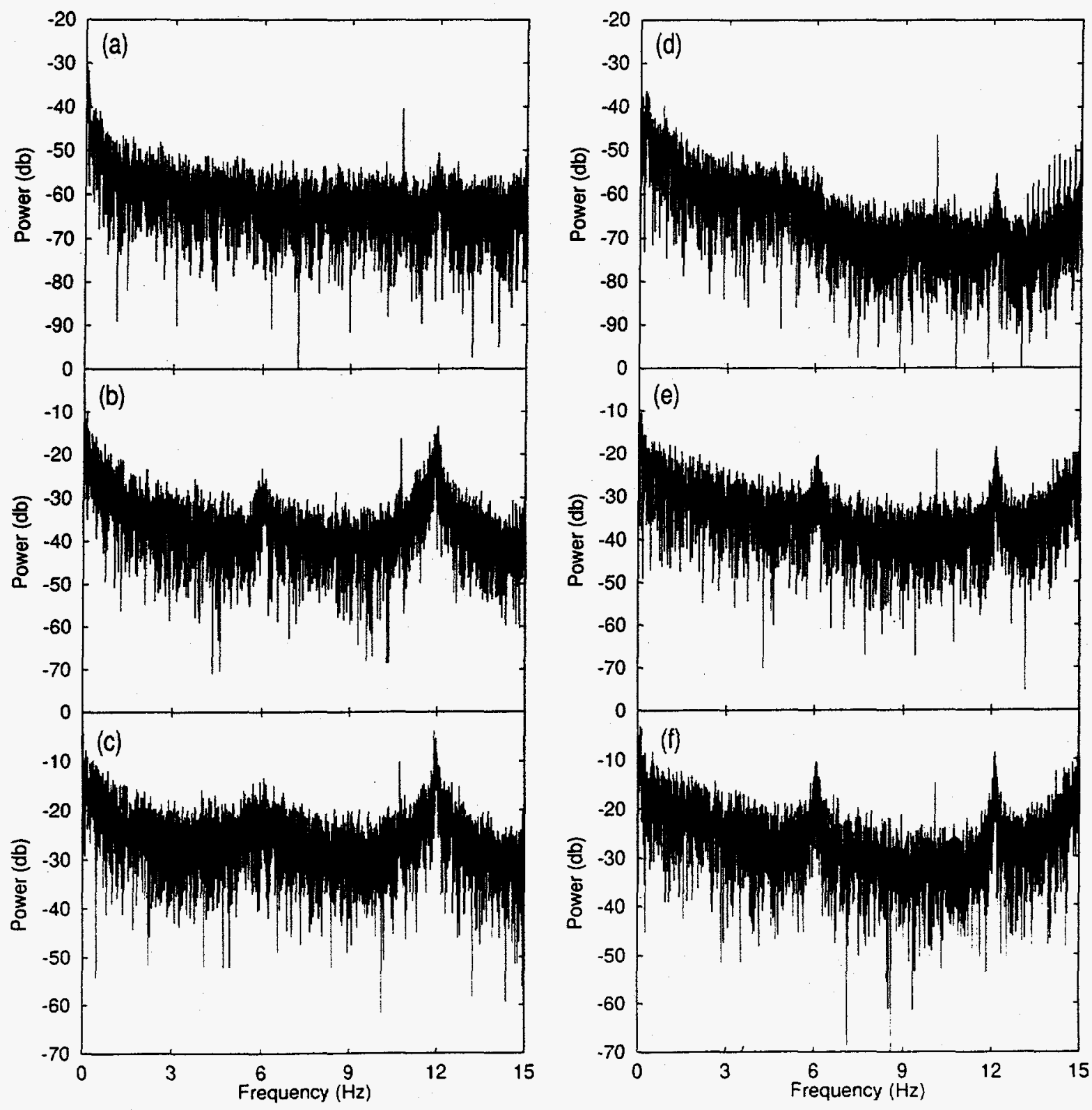

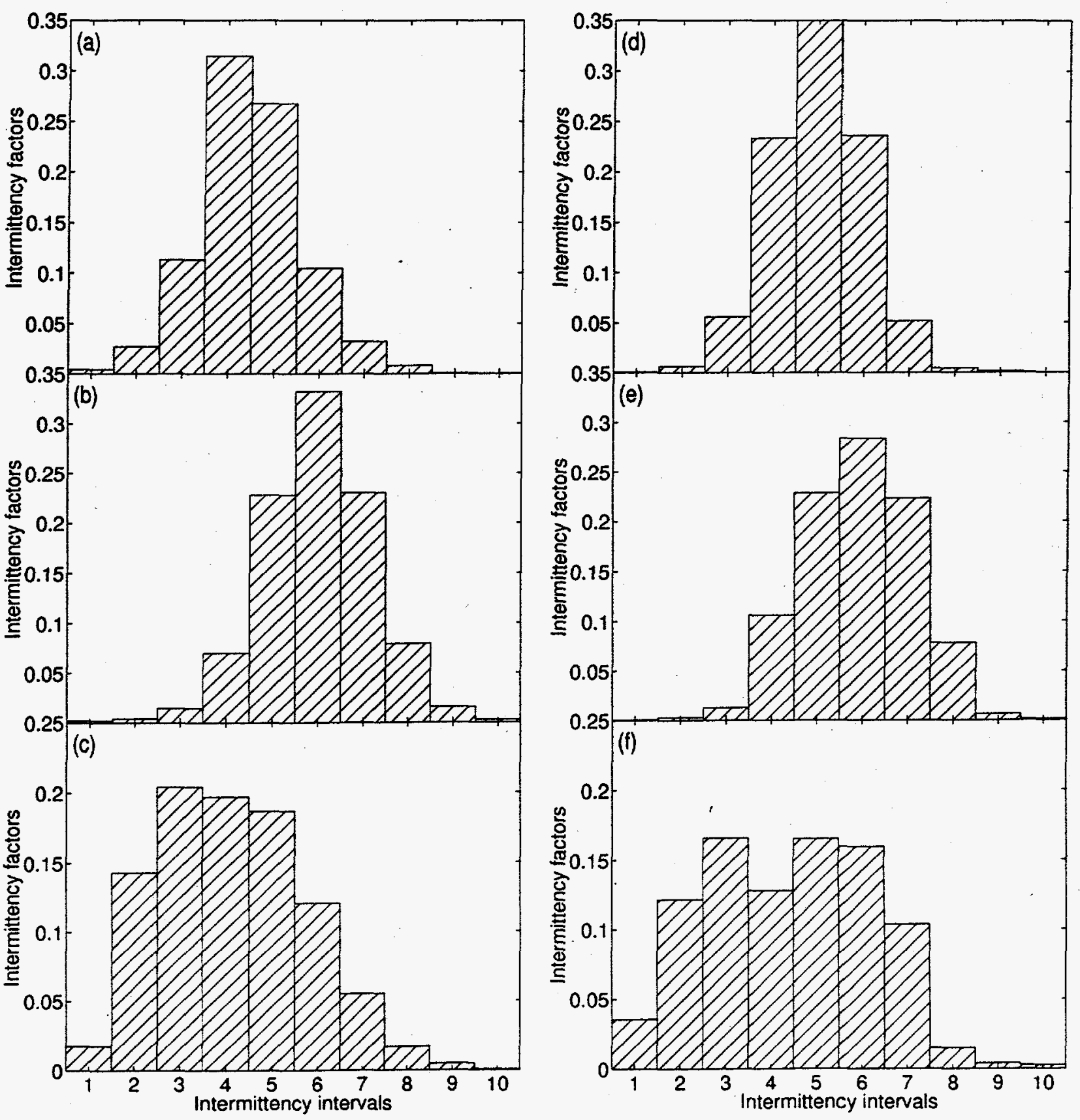

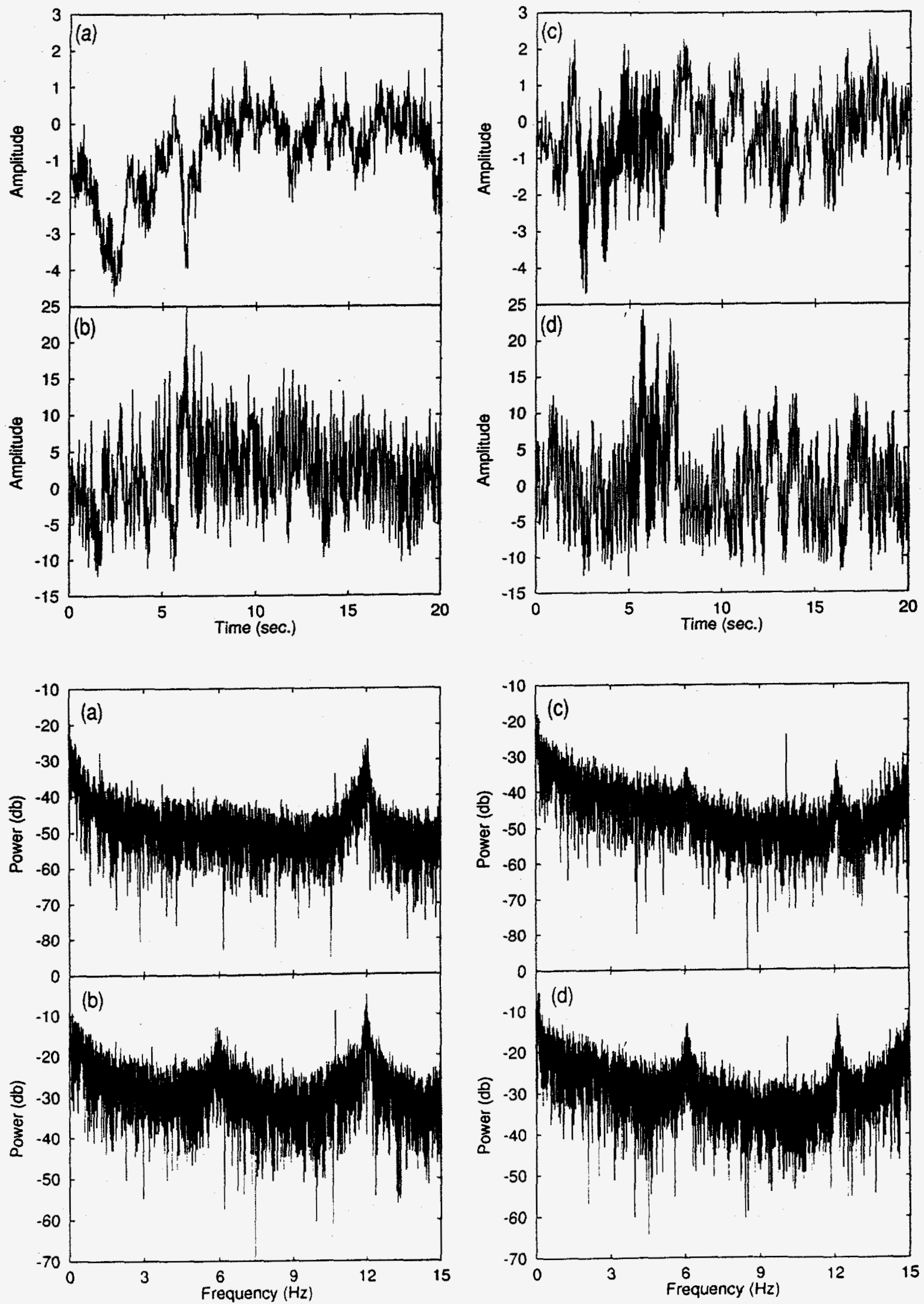

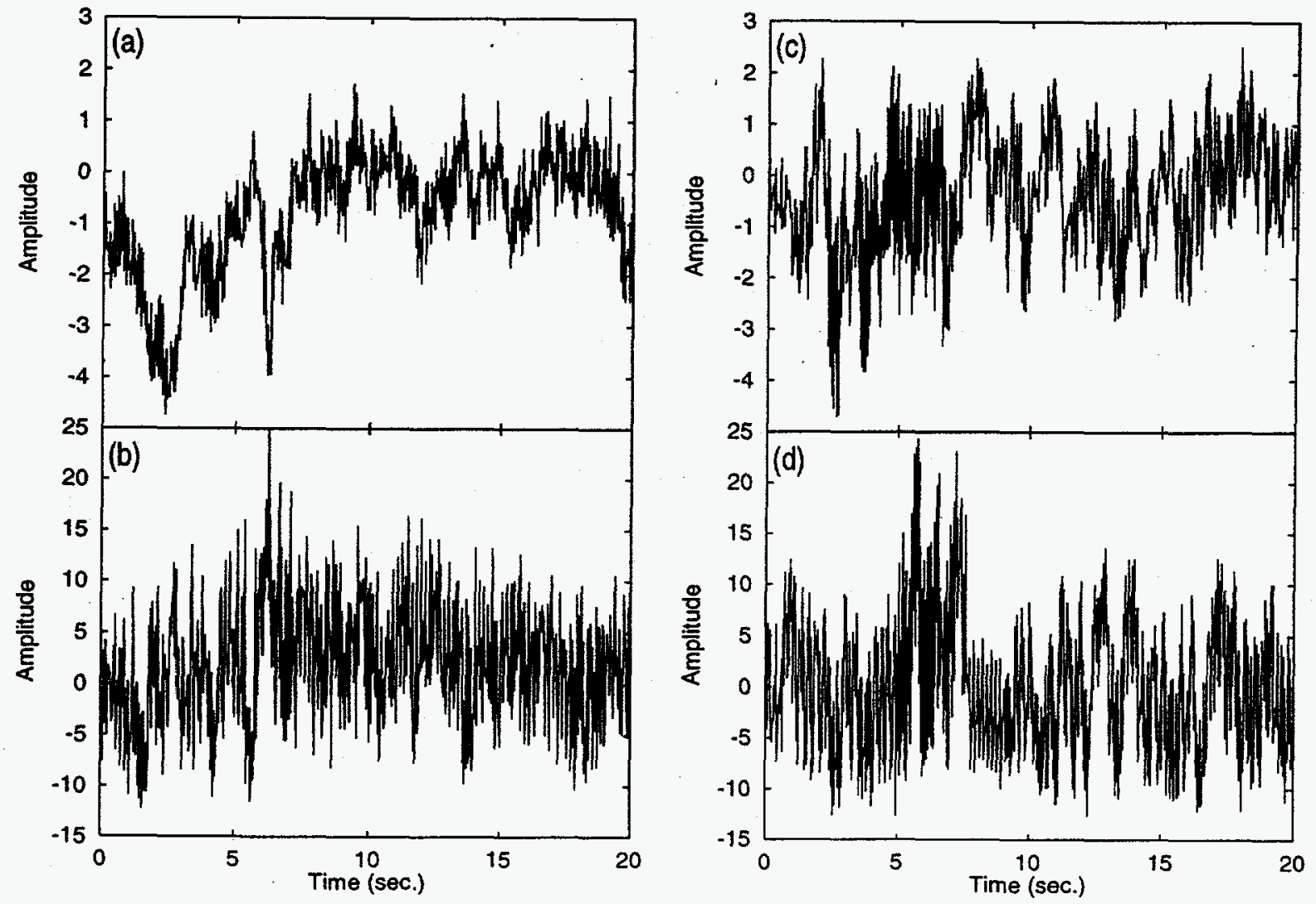

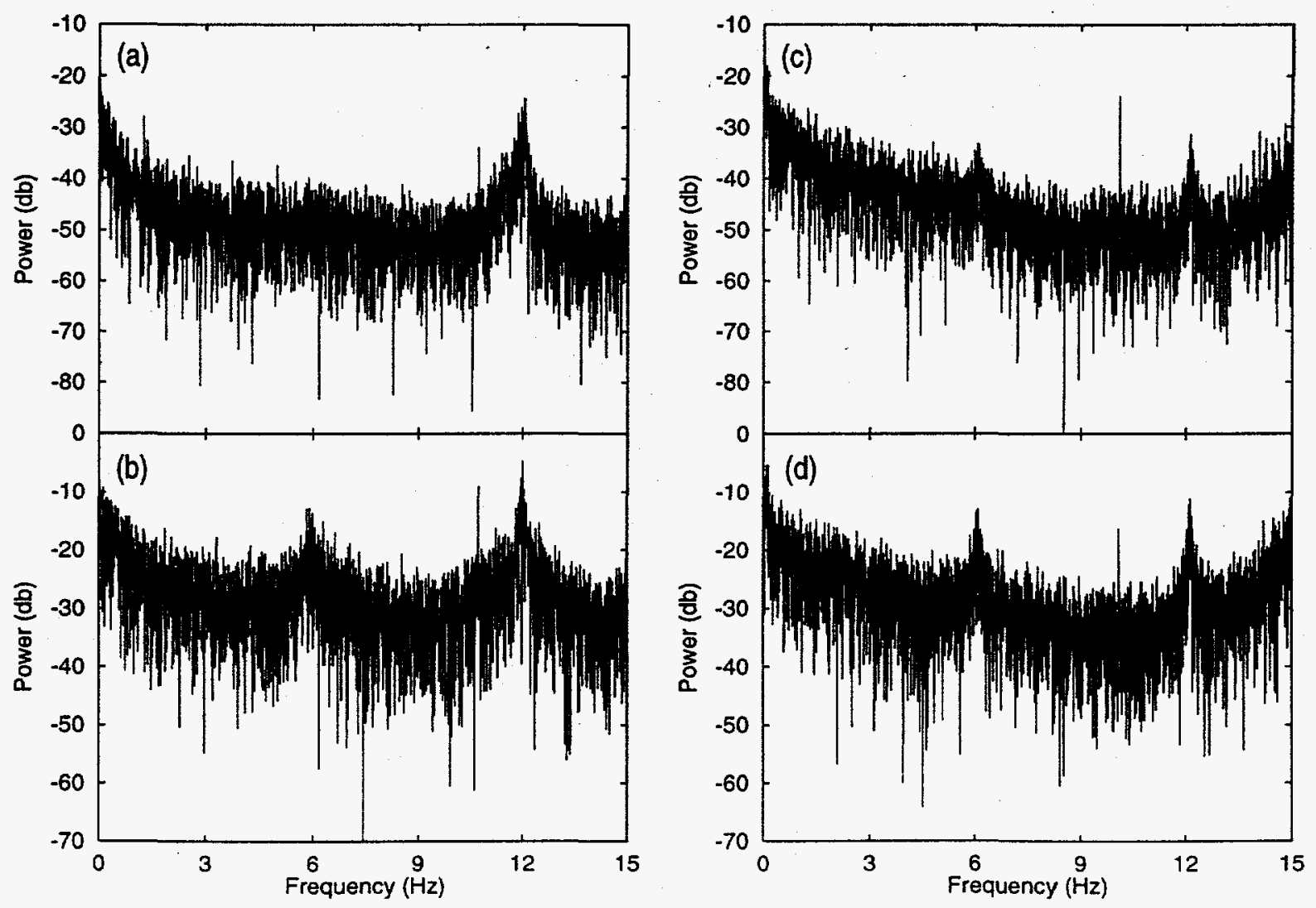

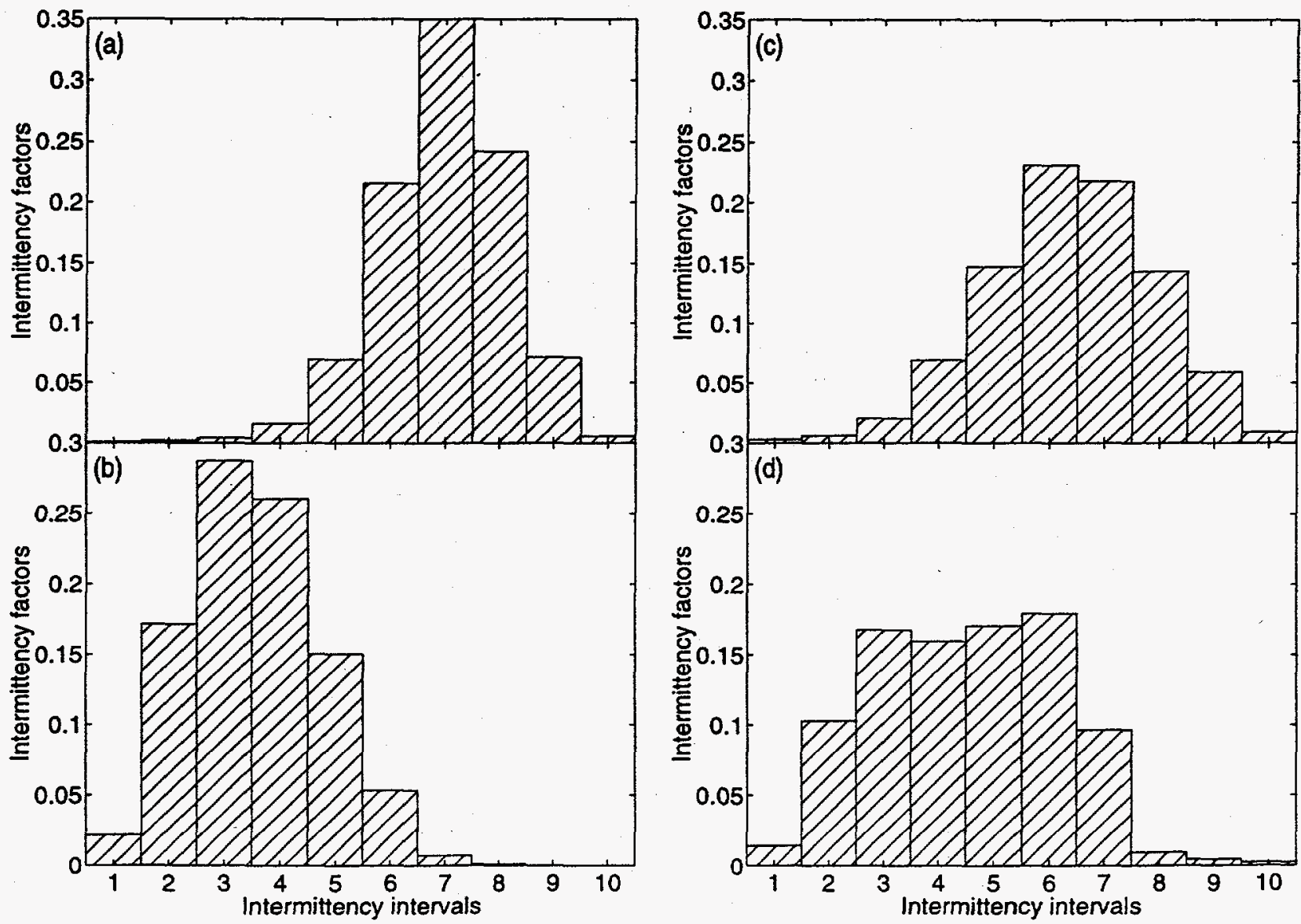